Case Report Journal of Epilepsy Research pISSN 2233-6249 / elSSN 2233-6257

Received January 10, 2016

Accepted March 14, 2016

Corresponding author: Dae-Won Seo

Department of Neurology, Samsung Medical Center, Sungkyunkwan University School of Medicine, 81 Irwon-ro, Gangnam-gu, Seoul 06351, Korea

Tel. +82-2-3410-3595

Fax. +82-2-3410-0052

E-mail; daewon@skku.edu

\title{
Intracranial Cortical Calcifications in a Focal Epilepsy Patient with Pseudohypoparathyroidism
}

\author{
Ye Sel Kim', Jihyung Park' ${ }^{1}$, Yoonkyung Park², KyoungJin Hwang ${ }^{3}$, Dae Lim Koo ${ }^{4}$, Daeyoung \\ $\mathrm{Kim}^{5}$, Dae-Won Seo ${ }^{1,6}$ \\ ${ }^{1}$ Department of Neurology, Samsung Medical Center, Sungkyunkwan University School of Medicine, Seoul; \\ ${ }^{2}$ Department of Neurology, Konyang University College of Medicine, Daejeon; ${ }^{3}$ Department of Neurology, Kyung \\ Hee University School of Medicine; ${ }^{4}$ Department of Neurology, Seoul Metropolitan Government Seoul National \\ University Boramae Medical Center, Seoul; ${ }^{5}$ Department of Neurology, Chungnam National University School of \\ Medicine, Daejeon; ${ }^{6}$ Neuroscience Center, Samsung Medical Center, Seoul, Korea
}

\begin{abstract}
Patients with chronic parathyroid dysfunction often have intracranial calcification in deep gray matter (GM) and subcortical white matter (WM) of their brain. Some of them are also epilepsy patients. Although cortical etiologies are main cause of epileptic seizure, cortical calcification has not been reported in these patients. We report a newly diagnosed focal epilepsy patient whose brain magnetic resonance imaging revealed intracranial calcifications in cortical as well as subcortical areas. Blood lab revealed that he had hypocalcemia due to pseudohypoparathyroidism. Video EEG monitoring revealed the ictal EEG mainly consist of polymorphic delta to theta waves with maximum at right temporal area followed by background attenuation and muscle artifacts. The interictal EEG showed multiple focal spike-wave discharges. After given oral calcium and calcitriol supplement, his calcium and phosphorous level normalized and he remains seizure free. This is the first case to show cortical calcification in a patient with pseudohypoparathyroidism. Cortical calcification could be an important measure of seizure burden in these patients and thus sophisticated imaging protocols should be used to visualize the extent of calcium deposits. (2016;6:31-35)
\end{abstract}

Key words: Hypocalcemia, Epilepsy, Focal seizure, Intracranial calcification, Cortical calcification

\section{Introduction}

Epilepsy in hypocalcemic patients has been reported for long period but pathophysiology underlying the hypocalcemic seizure remains to be elucidated. There have been limited reports on their semiology or EEG findings. Hypocalcemia induce a generalized seizure by changing neuroexcitability and seizure threshold. ${ }^{1,2}$ In addition, atypical absence, atonic, and brief focal (partial) seizure are also reported in these patients. ${ }^{1,3}$ Previous EEG findings include slowing of background rhythm, diffuse increase in slow wave activities with frontal preponderance, increased fast activity and multifocal spikes. ${ }^{1,4}$

Up to $20-25 \%$ of patients with acute hypocalcemia present epileptic seizure and up to $70 \%$ of chronic hypocalcemia patients are associated with epilepsy. ${ }^{1}$ Common etiologies for chronic hypocalcemia are vitamin D deficiency and parathyroid hormone (PTH) dysfunction such as hypoparathyroidism (low PTH level) or pseudohypoparathyroidism (ineffective peripheral PTH receptor). ${ }^{2}$
In chronic parathyroid dysfunction, as many as fifty percent of the patients have intracranial calcification in deep GM and subcortical WM of their brain and some of them are also epilepsy patients. ${ }^{3}$ However, cortical calcification has not been demonstrated in these patients. In mid 1900s, researchers reported calcification in frontal or occipital cortices in hypocalcemic patients but their figures illustrated calcification in subcortical fibers rather than cortical GM with skull radiographs and one early computed tomography (CT) scan with limited resolution. ${ }^{3}$ What the researchers meant by cortex seemed cerebral lobes including WM fibers and the point of papers was that calcification outside BG also exists. There have been no studies with modern CT or MRI reporting cortical calcification in chronic hypocalcemia.

The relationship between cortical calcification and epileptic seizure had been demonstrated in other brain disease such as neurocysticercosis or Sturge-Weber Syndrome (SWS). ${ }^{5}$ Here, we report a focal seizure patient with pseudohypoparathyroidism, who had intracranial calcification in cortical as well as subcortical areas. 
Table 1. Summary of biochemical laboratory findings

\begin{tabular}{|c|c|c|c|}
\hline Tests & Initial & 3 months after treatments & Normal range \\
\hline \multicolumn{4}{|l|}{ CBC } \\
\hline WBC $\left(\times 10^{3} / \mu \mathrm{L}\right)$ & 7.01 & & $3.8-10.6$ \\
\hline $\mathrm{Hb}(\mathrm{g} / \mathrm{dL})$ & 15.3 & & $13-17$ \\
\hline Platelet $\left(\times 10^{3} / \mu \mathrm{L}\right)$ & 258 & & $141-316$ \\
\hline \multicolumn{4}{|l|}{ Electrolyte } \\
\hline Sodium (mmol/L) & 143 & & $136-145$ \\
\hline Potassium (mmol/L) & 4.1 & & $3.5-5.1$ \\
\hline Chloride (mmol/L) & 96 & & $1.9-2.5$ \\
\hline Magnesium (mg/dL) & 2 & 2.3 & $1.3-2.5$ \\
\hline Calcium (mg/dL) & 5 & 7.8 & $8.0-10.4$ \\
\hline Ionized calcium (mmol/L) & 0.67 & 1.05 & $1.05-1.35$ \\
\hline Phosphate (mg/dL) & 6.3 & 4.5 & $2.5-4.5$ \\
\hline \multicolumn{4}{|l|}{ Hormones } \\
\hline PTH (pg/m) & 114 & 187.4 & $15-68$ \\
\hline D, 25-hydroxy (ng/mL) & 12.5 & 15.8 & $<20$ \\
\hline 25-hydroxyvitamin D2 (mg) & $<3$ & $<3$ & \\
\hline 25-hydroxyvitamin D3 (mg) & 12.5 & 15.8 & \\
\hline \multicolumn{4}{|l|}{ Liver function profile } \\
\hline AST & 26 & & $0-40$ \\
\hline ALT & 13 & & $0-40$ \\
\hline Alkaline phosphatase (U/L) & 83 & 83 & $40-130$ \\
\hline
\end{tabular}

CBC, complete blood count; WBC, white blood cell; Hb, hemoglobin; PTH, parathyroid horomon; AST, aspartate transaminase; ALT, alanine transaminase.

\section{Case}

36 year old male came to our clinic for evaluation of seizure episodes which started two years ago. His parents witnessed him stop talking in the conversation and staring blank 3 to 4 times a day. Initially his symptom was subtle to be noticed but later his symptom became concrete to be recognized by the patient himself. The frequency of complex partial seizures was 3 to 4 times a day. He was given levetiracetam $500 \mathrm{mg}$ a day for two weeks which only had partial response. We admitted him for epilepsy evaluation. Previous his medical records included a pre-term born and a laser ablation of bilateral cataracts at the age of thirty three years old. He had no family history of any endocrine or neurologic diseases. Clinical examination did not reveal any physical abnormalities associated with Albright-hereditary osteodystrophy or rickets. The neuropsychological tests revealed mild learning and memory impairment although his score of full scale IQ was 105.

His blood lab showed low calcium level $5 \mathrm{mg} / \mathrm{dL}$ (normal range: 8.0-10.4 mg/dL) and high parathyroid hormone $114 \mathrm{pg} / \mathrm{mL}$ (normal range: $15-68 \mathrm{pg} / \mathrm{mL}$ ) and phosphorous level $6.3 \mathrm{mg} / \mathrm{dL}$ (normal range: $2.5-4.5 \mathrm{mg} / \mathrm{dL}$ ). Additional parameters measured are listed in Table 1. CT (Fig. 1A) and T1 magnetic resonance imaging (MRI) (Fig. 1B) scan of his brain revealed bilaterally symmetrical calcification in basal ganglia (BG), thalami, cerebellar hemispheres and subcortical WM of bilateral frontoparietal lobes. In addition, calcifications along left insula and bilateral occipital cortical ribbon were present.

During video-EEG monitoring, he had 6 seizures which usually started when he made a sudden movement. He felt abnormal sensation of right leg followed by behavior arrest, unresponsiveness and dystonia of left arm. Brief myoclonic jerk then followed and his head turned toward right. The ictal EEG changes were initiated (Fig. 2A, B) with polymorphic delta $(2-3 \mathrm{~Hz}$ ) wave at right hemisphere, which showed maximum activity in right temporal region. Background attenuation followed then muscle artifact appeared as the patient's left arm became dystonic. Semirhythmic delta wave reappeared at right hemisphere, the rhythmic delta activity continued to rhythmic theta wave and became beta wave at the end of the ictal rhythm. The interictal EEG (Fig. 2C) showed multifocal spikes shown mainly over left frontotemporal (51.1\%) over right frontotemporal hemisphere 

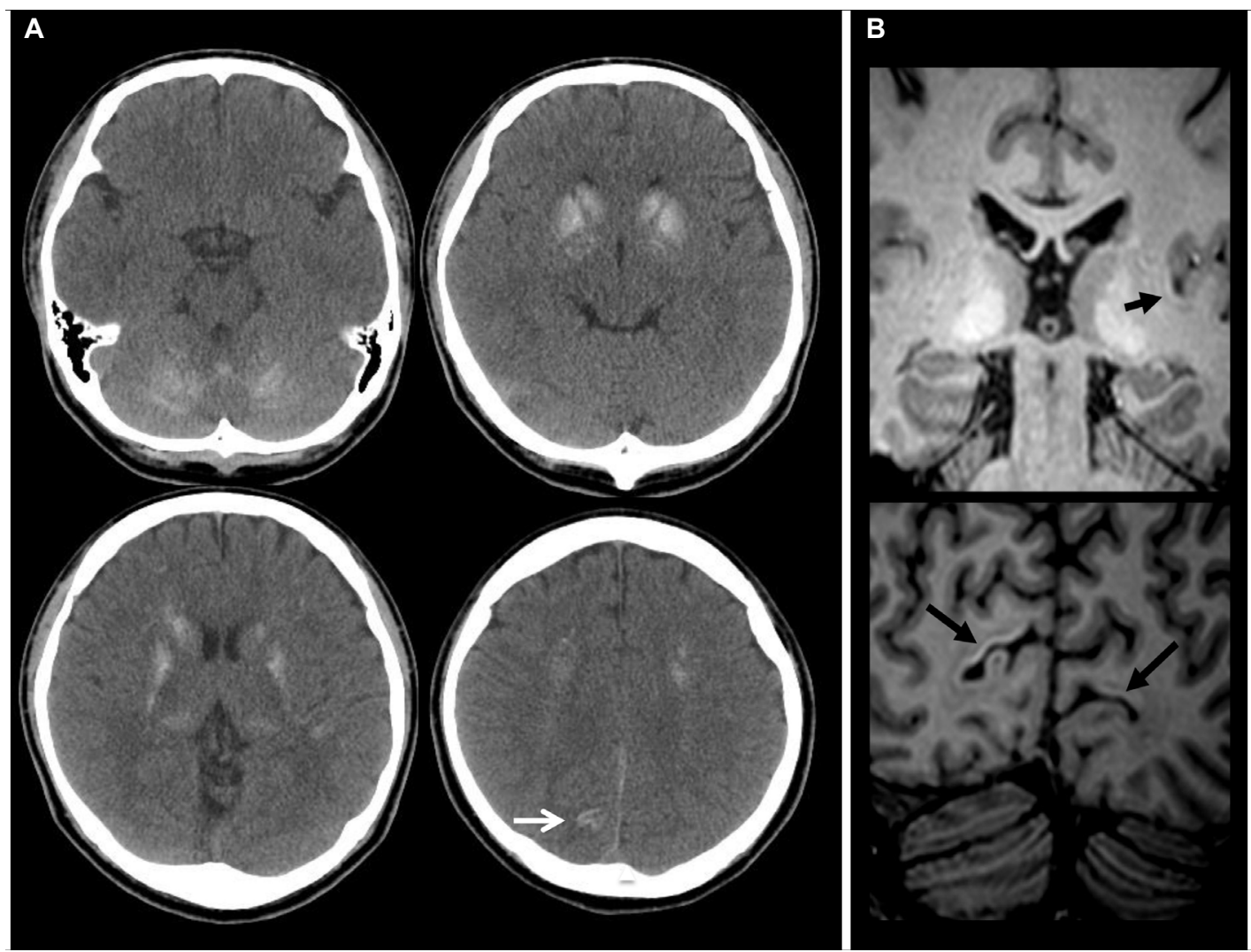

Figure 1. Neuroimages of the patient. Precontrast brain CT (A) reveals bilateral symmetric calcifications in cerebellar hemisphere, basal ganglia, thalami, subcortical white matter and cerebral cortex (arrow). Cerebral cortical lesion makes "W-shaped" cortical ribbon. Brain MRI (B) reveals linear high signal intensities in left insular cortex (short arrow) and both occipital cortical areas in non-enhanced T1 coronal imagine (long arrows).
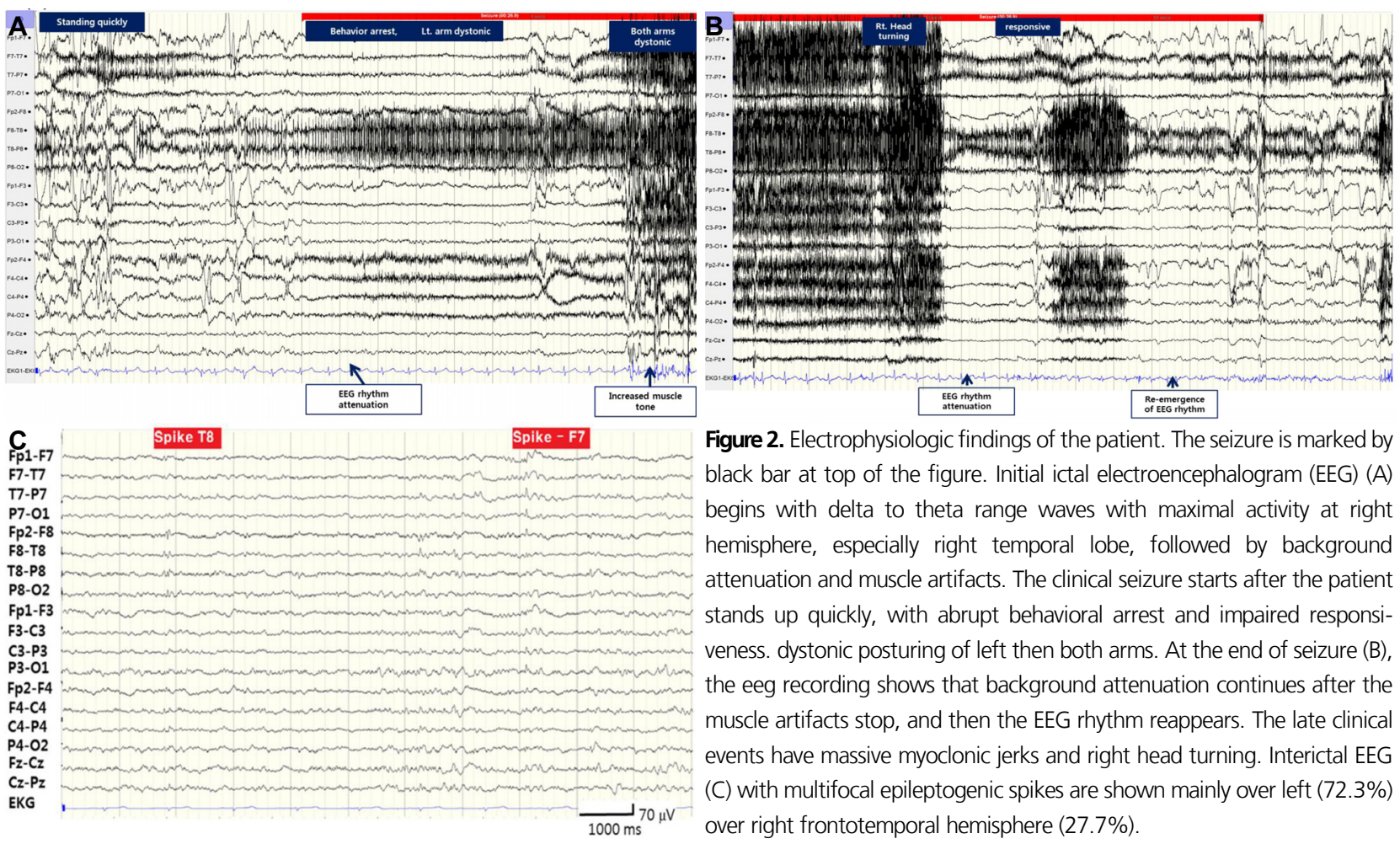

Figure 2. Electrophysiologic findings of the patient. The seizure is marked by black bar at top of the figure. Initial ictal electroencephalogram (EEG) (A) begins with delta to theta range waves with maximal activity at right hemisphere, especially right temporal lobe, followed by background attenuation and muscle artifacts. The clinical seizure starts after the patient stands up quickly, with abrupt behavioral arrest and impaired responsiveness. dystonic posturing of left then both arms. At the end of seizure (B), the eeg recording shows that background attenuation continues after the muscle artifacts stop, and then the EEG rhythm reappears. The late clinical events have massive myoclonic jerks and right head turning. Interictal EEG (C) with multifocal epileptogenic spikes are shown mainly over left (72.3\%) over right frontotemporal hemisphere (27.7\%). 
(27.7\%).

After monitoring, levetiracetam $500 \mathrm{mg}$ per day was restarted with supplement of calcium carbonate $1,500 \mathrm{mg}$ and calcitriol 0.25 mcg per day. He became seizure free while his calcium and phosphorous levels almost normalized at the three months follow up.

\section{Discussion}

In this case, we described a focal epilepsy patient with pseudohypoparathyroidism and intracranial calcification. Although hypocalcemia alone can cause epileptic seizure, focal metabolic seizure is rare. Thus we hypothesized that his unusual distribution of calcification in cortical ribbon were related to the epileptogenicity of his partial seizure.

By EEG-video monitoring, we attempted to localize the epileptogenic focus of his seizure. Interictal spikes were multifocal in nature and predominant over left $(72.3 \%)$ versus right (27.7\%) frontotemporal regions. This could be result of the low serum calcium level but also of the cortical calcium deposit as left temporal spikes were most frequently seen correlated with left temporal calcification. During the ictal phase, the seizure started with right somatosensory aura followed by left limb dystonic posturing and right head turning and staring. The somatosensory aura in right leg suggests the ictal symptomatogenic areas could be located more cortically than subcortically. Somatosensory aura is known to arise mostly from contralateral postcentral gyrus but also sometimes from ipsilateral secondary somatosensory area (SSA) or temporal cortices. ${ }^{6,7}$ According to previously studied semiology localization, succeeding left limb dystonic posturing accompanied by right head turning suggest the ictal focus is in the right hemisphere propagated to the right BG. ${ }^{6}$ Thus, it is likely that his aura originated from right hemisphere, either SSA or temporal area, and spread to ipsilateral BG. The ictal EEG findings of delta to theta waves with maximal activity at the right hemisphere, especially right temporal lobe, also support this assumption. Unfortunately, his CT and T1 MRI showed calcification most clearly in left temporal and bilateral occipital cortices, not right temporal or parietal cortices. Intracranial calcifications with calcium concentration of $30 \%$ or less will cause $\mathrm{T} 1$ hyperintensity but at higher concentration, the intensity of the signal diminishes. ${ }^{8}$ Gradient echo protocol was also done but thicker sections and blooming artifact at basal cerebral surface made it harder to visualize calcification than T1 MR or CT imaging. Susceptibility Weighted Imaging (SWI), relatively recent MR protocol, has been recognized as a superior tool in visual- ization of calcification and SWI protocol was employed to demonstrate the calcified cortical lesion in other epileptic disease. For example, Sturge-Weber Syndrome patients with severely calcified cortex demonstrated by SWI, suffered from a higher seizure burden (a measure of seizure frequency and epilepsy duration) and had a trend for earlier seizure onset. ${ }^{5}$ Unfortunately we did not include SWI protocol when we took our patient's MRI.

Whether intracranial calcification in other deep structure, especially $B G$, contributes to the epileptogenicity of these hypocalcemic epilepsy patients is unknown. Since epilepsy is known to be cortical origin, BG calcification sparing cortices has been disregarded in understanding epileptogenicity of chronic hypocalcema. However, there are studies which demonstrated the role of BG in epilepsy propagation and modulation. Some modeled BG as a seizure propagation pathway by injection of different NMDA antagonist into the substantia nigra pars reticulate suppressing both behavioral and EEG expression of epileptic seizures. ${ }^{9-11}$ Others suggested BG as a remote inhibitory circuit of epileptic seizure. ${ }^{12,13}$ Calcium deposit in BG may disrupt an inhibitory circuit and make patients with hypocamesia vulnerable to epileptic seizure. Functional imaging such as Single-photon emission computed tomography (SPECT) or positron emission tomography (PET) might be able to demonstrate functional compromise of $B G$ by the mineral deposit.

Despite the apparent absence of familial history and without a confirmatory genetic or molecular test, the combination of biochemical abnormality, neuroimaging finding, clinical course (early cataracts, epilepsy, and treatment response) assured us the diagnosis of pseudohypoparathyroidism as the etiology for his chronic hypocalcemia. Hypocalcemia and hyperphosphetemia with high level of PTH and low level of 25-hydroxyvitamin D can be also seen in secondary hyperparathyroidism due to vitamin $D$ deficiency. Although, vitamin D deficiency can cause hypocalcemic seizures in infants and young children ${ }^{1,14}$, adult with such severe hypocalcemia due to vitamin D deficiency and consequently epileptic seizure or intracranial calcification is very rare. Furthermore, at three months follow up, his PTH level increased regardless of calcitriol supplement. If his hypocalcemia and hyperparathyroidism are secondary due to vitamin D deficiency, calcitriol will increase serum calcium level and consequently decrease PTH level unlike his case.

In summary, we found that in addition to classical subcortical location, cortical calcification can be seen in chronic hypocalcemia due to pseudohypothyroidism. The electroclinical finding showed that the patient's seizure arise from right temporal lobe, although the cortical 
calcification was most clearly demonstrated in left insular and bilateral occipital cortices. Thus, we were not able to demonstrate that these cortical deposits are directly related to epileptogenicity. However, important observations were made. Multifocal interictal spikes were seen dominantly seen over left temporal area where the mineral deposit was clearly seen. Also, during ictal phase, EEG change was mainly initiated in unilateral (right) hemisphere suggesting focal metabolic seizure originate from unilateral hemisphere as seen in other focal epilepsy. His semiology suggests possible seizure propagation to subcortical structure especially BG. Therefore, subcortical calcification commonly seen in chronic hypocalcemia patients might contribute to epilepsy propagation as well. Lastly, even in focal epilepsy patients, neuronal hyperexcitability theory is important as we noted that his seizures which initially did not respond to conventional antiepileptic drug became quickly controlled with calcium and calcitriol supplement. This could be attributed to the stability of neuronal cells by correction of hypocalcemia.

Our limitation was that we did not include more sophisticated imaging protocols like SWI in visualization of cortical calcification in our patient. Visualization of intracranial calcification in chronic hypocalcemia patients by neuroimaging will enable us to understand the nature of hypocalcemic epilepsy better and this could be a possible measure of seizure burden and prognosis in these patients.

\section{References}

1. Nardone R, Brigo F, Trinka E. Acute symptomatic seizures caused by electrolyte disturbance. J Clin Neurol 2016;12:21-33.

2. Carroll R, Matfin G. Endocrine and metabolic emergencies: hypocalcaemia. Ther Adv Endocrinol Metab 2010;1:29-33.

3. Bloom RA, Rubinger D, Pogrund H. Frontal lobe calcification in hypo- parathyroid states. J Neurol 1983;229:65-8.

4. Dickson LG, Morita Y, Cowsert EJ, Graves J, Meyer JS. Neurological, electroencephalographic, and heredo-familial aspects of pseudohypoparathyroidism and pseudopseudohypoparathyroidism. J. I Neurol Neurosurg Psychiatry 1960;23:33-9.

5. Wu J, Tarabishy B, Hu J, et al. Cortical calcification in Sturge-Weber Syndrome on MRI-SWl: relation to brain perfusion status and seizure severity. J Magn Reson Imaging 2011;34:791-8.

6. Foldvary-Schaefer N, Unnwongse K. Localizing and lateralizing features of auras and seizures. Epilepsy Behav 2011;20:160-6.

7. Tuxhorn IE. Somatosensory auras in focal epilepsy: a clinical, video EEG and MRI study. Seizure 2005;14:262-8.

8. Ginat DT, Meyers SP. Intracranial lesions with high signal intensity on T1-weighted MR images: differential diagnosis. RadioGraphics 2012 32:499-516.

9. Bouilleret $V$, Semah F, Biraben A, et al. Involvement of the basal ganglia in refractory epilepsy: an 18F-fluoro-L-DOPA PET study using 2 methods of analysis. J Nucl Med 2005;46:540-7.

10. Deransart C, Lê-Pham BT, Hirsch E, Marescaux C, Depaulis A. Inhibition of the substantia nigra suppresses absences and clonic seizures in audiogenic rats, but not tonic seizures: evidence for seizure specificity of the nigral control. Neuroscience 2001;105:203-11.

11. Shehab $S$, Simkins $M$, Dean $P$, Redgrave P. The dorsal midbrain anticonvulsant zone--III. Effects of efferent pathway transections on suppression of electroshock seizures and defence-like reactions produced by local injections of bicuculline. Neuroscience 1995;65:697-708.

12. Dematteis $M$, Kahane $P$, Vercueil $L$, Depaulis $A$. MRI evidence for the involvement of basal ganglia in epileptic seizures: an hypothesis. Epileptic Disord 2003;5:161-4.

13. Biraben A, Semah F, Ribeiro MJ, Douaud G, Remy P, Depaulis A. PET evidence for a role of the basal ganglia in patients with ring chromosome 20 epilepsy. Neurology 2004;63:73-7.

14. Wallis K. Severe vitamin D deficiency presenting as hypocalcaemic seizures in a black infant at 45.5 degrees south: a case report. Cases J 2008;1:12. 